\title{
Skp2 knockout reduces cell proliferation and mouse body size: and prevents cancer?
}

\author{
Liang Zhu ${ }^{1}$ \\ Department of Developmental and Molecular Biology, and Medicine, Albert Einstein Cancer Research Center and Liver Research \\ Center, Albert Einstein College of Medicine, 1300 Morris Park Avenue, Bronx, NY 10461, USA \\ Cell Research (2010) 20:605-607. doi:10.1038/cr.2010.71; published online 25 May 2010
}

Attaching multiple ubiquitins (a 76residue protein ubiquitously expressed in eukaryotic cells) covalently to a protein labels that protein for degradation in the proteasome (a large tunnel-like complex considered as a protein degradation factory). There are many types of ubiquitin ligases (the enzymes that carry out the protein ubiquitination reactions); one of them is the Culin-RING ubiquitin ligase (CRL). There are six Culin proteins and two RING proteins, forming six general types of Culin-RING core platforms for various substraterecruiting subunits to complete the formation of substrate-specific CRLs. The SCF type CRL is formed with Rbx 1 (a RING protein), $\underline{\mathrm{S}} k p 1, \underline{\mathrm{C} u l 1, \text { and an }}$ F-box protein. F-box proteins use their F-box sequences to bind Skp1, which, in turn, brings the F-box protein to Cull. There are about seventy F-box proteins in humans; one of them is Skp2. The name of the specific F-box protein is written in superscript after SCF as in $\mathrm{SCF}^{\mathrm{Skp} 2}$. The best-studied substrate of $\mathrm{SCF}^{\mathrm{Skp} 2}$ is the cyclin-dependent kinase inhibitor p27Kip1. For $\mathrm{SCF}^{\mathrm{Skp} 2}$ to mediate $\mathrm{p} 27$ ubiquitination, p27 must first be phosphorylated on Thr187 (T187) and an auxiliary protein $\mathrm{Cks} 1$ must also be present. This reaction therefore can be called the $\mathrm{SCF}^{\mathrm{Skp} 2 / \mathrm{kss}}$-p27T187p mecha-

Correspondence: Liang Zhu

E-mail: liang.zhu@einstein.yu.edu nism; X-ray crystal structure of this complex shows the structural requirement for phosphorylation of p27T187 [1]. In addition to $\mathrm{p} 27$, Skp2 has been reported to mediate ubiquitination of about thirty other proteins [2].

Skp2 knockout mice ( $S k p 2^{-/}$mice) were generated to determine the physiological roles of Skp2 [3]. Mouse embryo fibroblasts (MEFs) derived from $S k p 2^{--}$embryos and a number of organs in $S k p 2^{-\leftarrow}$ mice contain increased levels of 27 protein, indicating that Skp2 indeed plays important roles in p27 protein degradation. Skp $2^{--}$MEFs proliferate extremely slowly in culture and $S k p 2^{--}$mice are about $25 \%$ smaller compared with $S k p 2^{+/+}$littermates, indicating that Skp2 has a required general proliferation-promoting function. These defects of $S k p 2^{-/}$MEFs and $S k p 2^{-/}$ mice can be corrected by combined knockout of p27, indicating that p27 is the major target of Skp2.

This Skp2-p27 relationship immediately suggested the following. As an inhibitor of cyclin-dependent kinases, p27 is a suspected tumor suppressor. Indeed, p27 protein levels are often reduced in cancer cells and greater $\mathrm{p} 27$ reductions often correlate with poorer prognoses. Since Skp2 counters p27 (by targeting it for degradation), Skp2 is suspected to be an oncoprotein. Indeed, Skp2 protein levels are often increased in cancer cells, especially in cells with reduced p27 protein, and higher Skp2 protein levels often predict poorer prognoses. p27 knockout or ectopic expression of Skp2 could promote tumorigenesis in a number of mouse tumor models.

The next unanswered question was whether Skp2 knockout could inhibit tumorigenesis. Since Skp2 knockout can inhibit normal proliferation ( $S k p 2^{-/}$ MEFs proliferate extremely poorly and $S k p 2^{--}$mice are significantly smaller), it is likely that oncogene-induced abnormal proliferation would also be inhibited in $S k p 2^{--}$MEFs and in $S k p 2^{--}$mice. On the other hand, $S k p 2^{--}$MEFs and $S k p 2^{--}$ mice also contain increased cyclin $\mathrm{E}$ proteins and enlarged nuclei; these properties would favor tumorigenesis.

In a recent article in Nature [4], Lin and colleagues directly addressed this question. They report dramatic data showing that, in response to various oncogenic signals (ectopic expression of an activated Ras, ectopic expression of Ras together with an adenovirus oncoprotein E1A, knockout of the tumor suppressor Pten, or knockout of the tumor suppressor ARF), Skp2 $2^{--}$MEFs proliferated even more slowly and, moreover, exhibited a robust cellular senescence response. Cellular senescence is a permanent form of cell proliferation arrest induced when repeated cell divisions erode chromosome ends or when cells are put under oncogenic stress [5]. Development of most forms of cellular senescence depends on two fundamental tumor suppressor pathways ARF-p53 
and $\mathrm{p} 16-\mathrm{pRb}$. Lin and colleagues show that $S k p 2^{--}$MEFs developed robust senescence fully independently of ARFp53. The authors went on to show that combining Skp2 knockout conferred impressive tumor resistance to $\mathrm{Pten}^{+/}$ mice, $\mathrm{ARF}^{-/-}$mice, and $\mathrm{Pten}^{-/-}$prostate gland. These dramatic findings convincingly identify Skp2 as a new and exciting cancer therapy target in the clinic, and raise important new questions in the lab for further study.

How many and what kinds of tumorigenic signals can be inhibited by Skp2 knockout? Lin and colleagues used Pten knockout and ARF knockout as oncogenic signals in their study, and $S k p 2^{--}$mice were found to be resistant to both. $S k p 2^{--}$MEFs were additionally resistant to Ras and E1A. In another study, Skp2 knockout blocked tumorigenesis following loss of the tumor suppressor $\mathrm{pRb}$, but did not inhibit tumorigenesis induced by the chemical carcinogen ENU [6]. Skp2 knockout also did not significantly inhibit Mycdriven lymphomagenesis [7]. Taken together, these findings indicate that Skp2 knockout may not provide a block to tumorigenesis in general. The effects of Skp2 knockout must therefore be experimentally determined for any given type of tumorigenesis. This may prove similar with the specific tumor blocking effects of cyclin D1 knockout [8].

If Skp2 plays essential tumorigenic roles under specific oncogenic signals, what are the molecular links between a specific oncogenic signal and Skp2? An example is the connection between pRb and Skp2. pRb can inhibit Skp2's ability to ubiquitinate $\mathrm{p} 27$ [9], promote ubiquitination and degradation of Skp2 by the APC ubiquitin ligase [10], and repress the expression of Skp2 via repressing E2F (since Skp2 is an E2F target gene [11]). Thus, loss of $\mathrm{pRb}$ led to activation of $\mathrm{SCF}^{\mathrm{Skp} 2}$ activity in mediating p27 ubiquitination. Knockout of Skp2 abolished this effect of pRb loss to block tumorigenesis [6]. Understanding the molecular links between a particular oncogenic signal and Skp2 should reveal more therapeutic targets to interfere with Skp2 activity.

How does Skp2 function when it is found to be required under certain oncogenic signals? Although $\mathrm{p} 27$ is a major target of Skp2, Skp2 also targets many other proteins for degradation and Skp2 has non-ubiquitination functions such as binding to cyclin A [12] and p300 [13]. Furthermore, how Skp2 mediates p27 degradation has actually not been clearly delineated since abolishing p27 phosphorylation at Thr187 prevented ubiquitination of the p27T187A mutant (in which Thr187 is changed to Ala, which cannot be phosphorylated) in test tubes but not in $\mathrm{p} 27^{\mathrm{T} 187 \mathrm{~A} / \mathrm{T} 187 \mathrm{~A}}$ mice [14]. Since there are currently no known mechanisms for Skp2 to mediate ubiquitination of the p27T187A mutant, how $S k p 2^{-/}$mice accumulate $\mathrm{p} 27$ protein is not clear.

However, p27 $7^{\mathrm{T} 187 \mathrm{~A} / \mathrm{T} 187 \mathrm{~A}}$ mice were able to block tumorigenesis like $S k p 2^{-/}$ mice following $\mathrm{Rb} 1$ loss, revealing that Skp2 functions in the $\mathrm{SCF}^{\text {Skp2 }}$ ${ }^{\text {Cks1 }}$-p27T187p mechanism to promote tumorigenesis following Rb1 loss [6]. It will be important to determine whether p2 $7^{\mathrm{T} 187 \mathrm{~A} / \mathrm{T} 187 \mathrm{~A}}$ mice are also resistant to tumorigenesis induced by Pten knockout or ARF knockout.

Lin and colleagues revealed an interesting link between Skp2 and Atf4, a transcription factor whose levels often increase during endoplasmic reticulum (ER) stress. Although Atf4 protein levels were increased in $S k p 2^{--}$MEFs with Pten knockout or ARF knockout, the increases did not appear to be due to protein stabilization. Furthermore, other markers of ER stress such as BiP and p-Perk did not increase at the same time. These findings may point to a new direction to study Skp2 function in averting cellular senescence.

Finally, targeting Skp2 to treat cancer has been proposed before, but has now become a more pressing goal with experimental evidence in hand that Skp2 plays essential roles in tumorigenesis induced by loss of tumor suppressors Pten, $\mathrm{ARF}$, and $\mathrm{pRb}$, which are frequent events in various human cancers. Lin and colleagues addressed this issue with an inhibitor for CRLs. For the CRL type ligases to be active, the Culin subunit must be modified by covalent attachment of a small protein called NEDD8. For this attachment, NEDD8 must first be activated by an NEDD8 activating enzyme (NAE) in an ATP dependent reaction. In 2009, Soucy and colleagues reported the identification of an NAE inhibitor called MLN4924, which effectively inhibited growth of xenograft tumors derived from human colon cancer cells (HCT-116) and lung cancer cells (H522 and Calu-6) [15]. Lin and colleagues now show that MLN4924 can inhibit growth of xenograft tumors formed with human prostate cancer cell line PC3, which lacks Pten and p53, with associated cellular senescence. Cautions must be excised in interpreting this data since MLN4924 inhibits all CRLs and, therefore, the observed effects may not be entirely attributable to inhibition of $\mathrm{SCF}^{\text {skp2 }}$.

The published X-ray crystal structure of $\mathrm{SCF}^{\mathrm{Skp} 2 / \mathrm{ks} 1}-\mathrm{p} 27 \mathrm{~T} 187 \mathrm{p}$ should provide a basis for rational design of Skp2specific inhibitors [1]. Here, a concern is that $\mathrm{p} 27^{\mathrm{T} 187 \mathrm{~A} / \mathrm{T} 187 \mathrm{~A}}$ mice do not phenocopy $S k p 2^{--/}$mice in the regulation of p27 protein levels, except in blocking pituitary tumorigenesis following loss of $\mathrm{Rb} 1$. More studies are needed to determine whether $\mathrm{p} 27^{\mathrm{T} 187 \mathrm{~A} / \mathrm{T} 187 \mathrm{~A}}$ mice could phenocopy $\operatorname{Skp} 2^{-/}$mice in other types of tumors that could be blocked by Skp2 knockout to identify types of cancers that would potentially be indicated for this treatment strategy. On the other hand, although targeting NAE is not specific for Skp2, it appears to work effectively for the tested tumor cells and is well tolerated by mice. Since many inhibitors that work effectively in labs only to fail spectacularly in clinics, scientists need to continue to explore and discover new ways to treat cancer. 


\section{References}

1 Hao B, Zheng N, Schulman BA, et al. Structural basis of the Cks1-dependent recognition of $\mathrm{p} 27$ (Kip1) by the SCF(Skp2) ubiquitin ligase. Mol Cell 2005; 20:9-19.

2 Frescas D, Pagano M. Deregulated proteolysis by the F-box proteins SKP2 and $\beta$-TrCP: tipping the scales of cancer. Nat Rev Cancer 2008; 8:438449.

3 Nakayama K, Nagahama H, Minamishima YA, et al. Targeted disruption of Skp2 results in accumulation of cyclin E and p27(Kip1), polyploidy and centrosome overduplication. EMBO J 2000; 19:2069-2081.

4 Lin HK, Chen Z, Wang G, et al. Skp2 targeting suppresses tumorigenesis by Arfp53-independent cellular senescence. Nature 2010; 464:374-379.

5 Campisi J, d'Adda di Fagagna F. Cellular senescence: when bad things happen to good cells. Nat Rev Mol Cell Biol 2007; 8:729-740.

6 Wang H, Bauzon F, Ji P, et al. Skp2 is required for survival of aberrantly proliferating Rb1-deficient cells and for tumorigenesis in $\mathrm{Rb}^{+/-}$mice. Nat Genet 2010; 42:83-88.

7 Old JB, Kratzat S, Hoellein A, et al. Skp2 directs Myc-mediated suppression of p27Kip1 yet has modest effects on Myc-driven lymphomagenesis. $\mathrm{Mol}$ Cancer Res 2010; 8:353-362.

$8 \mathrm{Yu}$ Q, Geng Y, Sicinski P. Specific protection against breast cancers by cyclin D1 ablation. Nature 2001; 411:1017-1021.

9 Ji P, Jiang H, Rekhtman K, et al. An RbSkp2-p27 pathway mediates acute cell cycle inhibition by $\mathrm{Rb}$ and is retained in a partial-penetrance $\mathrm{Rb}$ mutant. $\mathrm{Mol}$ Cell 2004; 16:47-58.

10 Binne UK, Classon MK, Dick FA, et al. Retinoblastoma protein and anaphasepromoting complex physically interact and functionally cooperate during cellcycle exit. Nat Cell Biol 2007; 9:225232.

11 Zhang L, Wang C. F-box protein Skp2: a novel transcriptional target of E2F. Oncogene 2005; 25:2615-2627.

12 Ji P, Goldin L, Ren H, et al. Skp2 contains a novel cyclin A binding domain that directly protects cyclin A from inhibition by p27Kip1. J Biol Chem 2006; 281:24058-24069.

13 Kitagawa M, Lee SH, McCormick F. Skp2 Suppresses p53-Dependent Apoptosis by Inhibiting p300. Mol Cell 2008; 29:217-231.

14 Malek NP, Sundberg H, McGrew S, et al. A mouse knock-in model exposes sequential proteolytic pathways that regulate $\mathrm{p} 27 \mathrm{Kip} 1$ in $\mathrm{G} 1$ and $\mathrm{S}$ phase. Nature 2001; 413:323-327.

15 Soucy TA, Smith PG, Milhollen MA, et al. An inhibitor of NEDD8-activating enzyme as a new approach to treat cancer. Nature 2009; 458:732-736. 